\title{
Experimental Analysis of a UAV-Based Wireless Power Transfer Localization System
}

\author{
Andrew Mittleider ${ }^{1}$, Brent Griffin ${ }^{1,2}$, and Carrick Detweiler ${ }^{1}$ \\ 1 Nebraska Intelligent MoBile Unmanned Systems (NIMBUS) Lab \\ Department of Computer Science and Engineering \\ University of Nebraska-Lincoln, Lincoln, NE 68588, USA \\ \{amittlei, carrick\}@cse.unl.edu \\ http://nimbus.unl.edu \\ 2 Department of Electrical Engineering and Computer Science, \\ University of Michigan \\ Ann Arbor, MI 48109, USA \\ bagriffin@gmail.com
}

\begin{abstract}
Sensors deployed in remote locations provide unprecedented amounts of data, but powering these sensors over long periods remains a challenge. In this paper, we develop and present a UAV-based wireless power transfer system. We discuss design considerations and present our system that allows a UAV to fly to remote locations to charge hard to access sensors. We analyze the impact of different materials on the wireless power transfer system. Since GPS does not provide sufficient accuracy, we develop and experimentally characterize a relative localization algorithm based on sensing the magnetic field of the power transfer system and optical flow that allows the UAV to localize the sensor with an average error of $15 \mathrm{~cm}$ to enable the transfer of on average $4.2 \mathrm{~W}$. These results overcome some of the practical challenges associated with wirelessly charging sensors with a UAV and show that UAVs with wireless power transfer systems can greatly extend the life of remotely deployed sensors.
\end{abstract}

\section{Introduction}

Wireless sensor networks are used in a wide range of applications from large scale terrestrial habitat monitoring [1] to underground [2] and underwater [3] systems because of their ability to measure a multitude of environmental variables with high frequency over long periods of time. Their ability to monitor these locations over long periods of time is significantly advancing science, however, powering these sensor networks remains a challenge despite advances in energy efficient sensor networks and battery technology. Current systems deployed for long periods either require additional infrastructure (e.g. power cables or solar panels) or periodic maintenance to replace batteries. We have developed a novel solution to this problem by using a UAV equipped with a resonant magnetic wireless power transfer system to charge sensors in hard-to-access locations [4]. The system can 


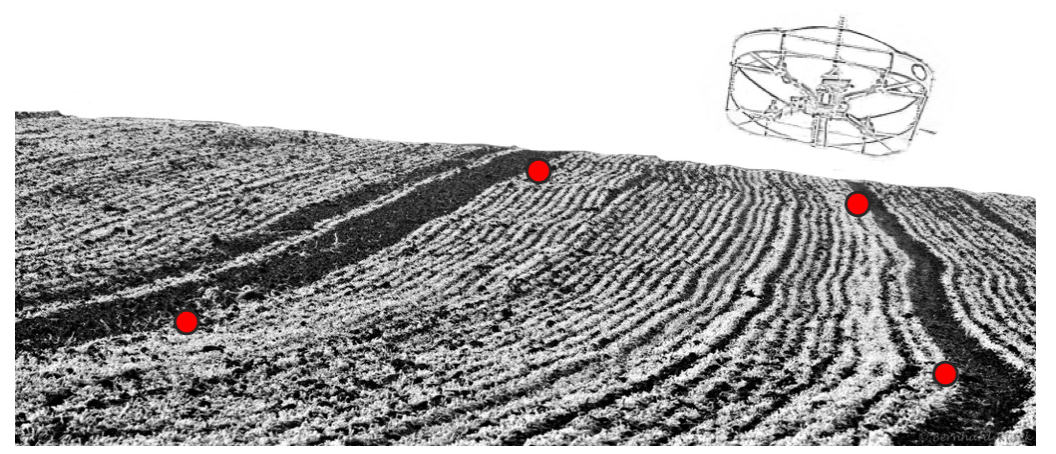

Fig. 1: Illustration showing a UAV charging WSN nodes embedded in a field.

transfer over 10 Watts at close distances and a range of about $1 \mathrm{~m}$. The system enables charging of sensors embedded in materials or in hard to access locations where physical charging connectors are impractical.

In this paper, we address the problem of getting the UAV close enough to the ground sensor to enable charging. We do this by developing a relative localization system based on sensing the magnetic field emitted by the wireless power transfer system and an optical flow camera. We use a weighted least-squares minimization approach based on the magnetic field model and empirical measurement estimations of the relative location. Precise relative localizing is critical since the positional error in the GPS that the UAV uses to navigate is too large to enable efficient charging. In addition, we want to avoid adding hardware to the UAV, as the additional payload would decrease flight time (e.g. adding a laser scanner to perform SLAM). The localization system we develop requires minimal additional hardware and we find experimentally that the average localization error in our system is $21 \mathrm{~cm}$, which yields an average power transfer rate of $4.2 \mathrm{~W}$. In addition, we investigate and show experimentally that the magnetic field sensing is not impacted by common materials non-magnetic materials such as dirt, woodchips, stone, and plastic. This means that sensors can be embedded, for instance in a field to monitor crops as shown in Fig. 1, and still be localized and charged.

\section{Related Work}

There are a variety of techniques that can be used to wirelessly transmit energy ranging from directed microwave energy [5] to radio frequency power harvesting [6]. We use magnetic resonance wireless to transfer power from our UAV. This is related to systems developed by Tesla over a century ago [7] and more recently researchers have shown that it is possible to transfer tens of Watts over 
medium distances (few meters). For instance, Kurs et al. transferred 60 Watts over 2 meters with $60 \%$ efficiency to power a light bulb [8]. Duong and Lee [9] use a variable coupling technique to improve power transfer efficiency at different ranges, and Sample and Meyer [10] significantly reduce wireless power inefficiencies by developing an algorithm that automatically tunes the drive frequency during over-coupling of the system. Instead of focusing on improving the efficiency of magnetically resonant wireless power transfer systems, our research focuses on obtaining precise relative localization between the UAV carrying the power transfer system and the sensor node being charged. We also examine the impact of embedding the sensors we are localizing and charging in different materials. Prior work by Seo et al. examined the impact of building materials such as softwood lumber, concrete brick, and drywall with insulation on wireless power transfer [11]. We add to this by also examining common outdoor materials and looking specifically at how these impact our magnetic resonant localization sensor.

Our work is inspired by Moore and Tedrake [12] who use a magnetic field to localize a glider UAV to perch on a power line. They use a standard magnetometer to sense the magnetic field from low-frequency, high-current power lines (40 Amps). They use a Kalman filter and track the magnetic field and eliminate positional ambiguity in the magnetic field sensors. They conclude that their method of localization is sufficient for a small UAV to perch on a power line, however, their system does not fully close the loop to enable control based on the sensor readings. Our work differs from theirs in a number of ways. First, our system operates at higher frequencies and lower currents so standard magnetometers do not work. Instead we develop a sensor that measures the voltage in a small resonant coil (see Section 4.2). Second, we are primarily interested in having a final position close enough to transfer power and are less interested in the trajectory during approach, which is critical to enable perching. Thus, we do not need to consider the details of vehicle dynamics, which allows us to easily adapt our approach for new vehicles. Finally, we demonstrate our system and perform full closed-loop control.

Also related to our work is methods for localizing based on radio transmissions. For instance, Tokekar et al. used bearing and signal strength to locate radio tagged fish with a robotic boat [13]. They use many wireless sensor network (WSN) nodes equipped with rotatable antenna to locate the fish. First, they map a relationship between the signal strength and the distance from a ground truth measurement. They fit a linear regression model of this data. When the radio strength is at its maximum value, the bearing and radio strength information is sent to a centralized computer. The position of the fish is then triangulated by creating an enclosed polygon with the bearing angles.

Finally, while we are one of the first to demonstrate charging of ground sensors from an aerial robot, the reverse problem of how to power an aerial vehicle was looked at in 1964 to wirelessly power a flying helicopter [14] and in 2011 was used to enable a 12 hour, record-length quad-rotor flight [15]. 


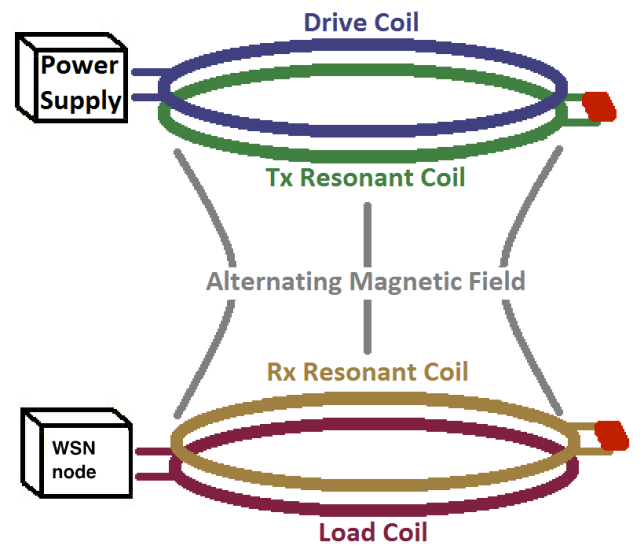

Fig. 2: Schematic for resonant power transfer.

\section{Power Transfer Design Considerations}

In this section we present background information on wireless magnetic resonant power transfer and discuss design considerations for UAV-based power transfer systems. Inductive power transfer involves at least two coils in close proximity sharing alternating magnetic fields. An alternating current (AC) in the transmitting coil produces a magnetic field that generates an alternating voltage in the receiving coil that can be applied to power or charge a device. Standard inductive power transfer is only efficient over short distances, but this limitation can be overcome with the use of strongly coupled magnetic resonances.

Power transfer is much more efficient over medium ranged distances by including two coupled resonant coils between the driven and loaded inductive coils, as seen in Fig. 2. In this configuration, the primary inductive coil, or Drive coil, is driven by an AC power supply. Due to the close proximity between the Drive coil and the first resonant coil, called the Tx resonant coil, oscillations occur and power is transferred to the Tx coil. The Tx coil causes the Rx coil to oscillate with a proportional degree of energy that is dependent on their coupling. The Tx and Rx coils do not have any direct load connected to them to interfere with the resonance. This allows them to couple and resonate over larger distances than is possible without resonant coils. The last coil, the Load coil, inductively receives power from the $\mathrm{Rx}$ coil in the same way that the Drive coil transfers energy to the Tx coil, and it applies the voltage that it gains across a load to receive the power.

The two primary factors that impact resonant wireless power transfer performance are the quality factor $(\mathrm{Q})$ of the coils and ensuring that all coils naturally resonate at a similar frequency. The quality factor represents how well a resonant coil can hold energy without losses to heat and is defined as [16]: 




Fig. 3: Coils with high quality factor (solid blue) can have lower coupling than those with lower quality factors (dashed red) due to manufacturing tolerances.

$$
Q=\frac{1}{R} \sqrt{\frac{L}{C}}
$$

where $R$ is the resistance of the coil $(\Omega), L$ is the inductance of the coil $(\mathrm{H})$, and $\mathrm{C}$ is capacitance (F). For details on how to compute these parameters see [17].

Intuitively, the quality factor can be thought of as how much energy a resonant system can hold compared to energy lost during a single cycle. However, as previously stated, while maximizing the quality factor is important when trying to minimize the losses within the coils, a high Q may have a detrimental effect. In practice, the resonant frequency of all of the coils will be slightly different from each other due to manufacturing imperfections and component tolerances. High $\mathrm{Q}$ factors cause a decrease in the bandwidth of the resonant coil, $\Delta f$ in Hz, which is defined as: $\Delta f=\frac{f_{r}}{Q}$.

If the resonant frequency of one coil is not within the bandwidth range of the other coil, they will couple poorly as shown in Fig. 3. The figure represents two sets of resonant coils. The solid blue curves represent coils which have a resonant frequency $f_{1}$ and $f_{2}$, respectively, and have a high $\mathrm{Q}$. The higher the curves intersect on the $y$-axis, the higher the power transfer. Notice that the high Q means that the peak power transfer is higher if they are aligned, but because of the difference in the coils natural resonant frequency (e.g. manufacturing tolerances), they are only able to transfer at about half of the optimal performance. The red, dashed curves show coils with the same resonate at the same frequencies $f_{1}$ and $f_{2}$, but lower $\mathrm{Q}$. If they were precisely aligned they would have lower overall transfer, but since they have higher bandwidth they better tolerate misalignment that results in higher power transfer in this configuration. While fixed, ground based power transfer systems can be precisely tuned, this can be difficult for UAV-based systems or other field robotics applications. For instance to be lightweight, our coils flex significantly during flight and further after rough landings or crashes their shape becomes distorted, changing their resonant frequencies. So while having high $\mathrm{Q}$ is typically good, too high of a $\mathrm{Q}$ may reduce performance in many robot systems. 


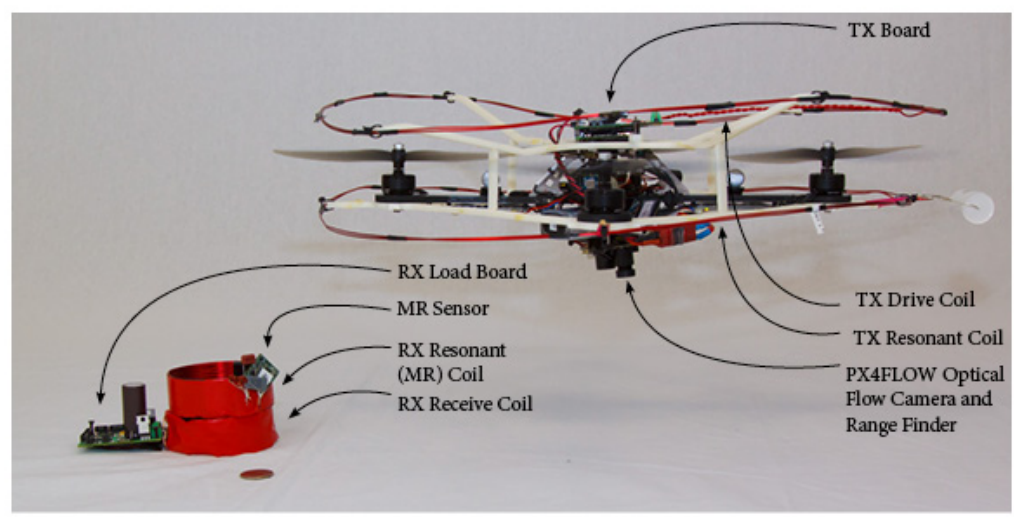

Fig. 4: The wireless power transfer system.

There are also a number of other considerations when designing resonant wireless power transfer systems. For low frequency systems energy losses are dominated by ohmic losses and high frequency losses by radiative losses [18]. The primary loss in our relatively low frequency system is ohmic, which causes the coils to heat when high currents move through the slightly resistive winds of the wire in each coil. In addition, we have switching losses in our AC power system. By decreasing the resistance, we can increase the $\mathrm{Q}$ and decrease the former losses. Unfortunately this may cause an increase in the switching losses due to higher currents moving through MOSFETs. Further, while using a thicker gauge wire is an easy way to decrease resistance and increase Q, it also adds weight to the UAV. Similar trade-offs must be made when adjusting the capacitance and inductance in the system to attempt to maximize the quality factor.

Now that we have briefly examined the theory behind magnetic resonant wireless power transfer and discussed design considerations, we go on to look at the specific components of our UAV-based wireless power transfer system and wireless sensor network node.

\section{System Components}

This section describes the hardware of the wireless power transfer system and the UAV. Fig. 4 shows an overview of the wireless power transfer system on the UAV. The system consists of a UAV, the wireless power transmitter on the UAV, and the receiving wireless sensor node.

\subsection{UAV Power Transfer System}

Fig. 4 shows an overview of the wireless power transfer system. On the UAV, the TX Drive Board sends an alternating current through the Drive Coil causing an alternating magnetic field that drives the neighboring Tx Resonant Coil. The Tx 


\begin{tabular}{|c|c|c|}
\hline Description & Variable & Value \\
\hline Coil Length & $l$ & $1.47655 \mathrm{~m}$ \\
Coil Radius & $r$ & $0.235 \mathrm{~m}$ \\
Resistance & $R$ & $0.0143 \Omega$ \\
Number of Wraps & $N$ & 2 \\
Inductance & $L$ & $5.20068 \times 10^{-} 6 \Omega$ \\
Capacitance & $C$ & $1.5 \times 10^{-} 7 \mathrm{~F}$ \\
Frequency & $f_{r}$ & $167 \mathrm{kHz}$ \\
Bundle Thickness & $c$ & $0.004 \mathrm{~m}$ \\
Quality Factor & $\mathrm{Q}$ & 411 \\
\hline
\end{tabular}

Table 1: Power transfer system parameters for the UAV and WSN node coils.
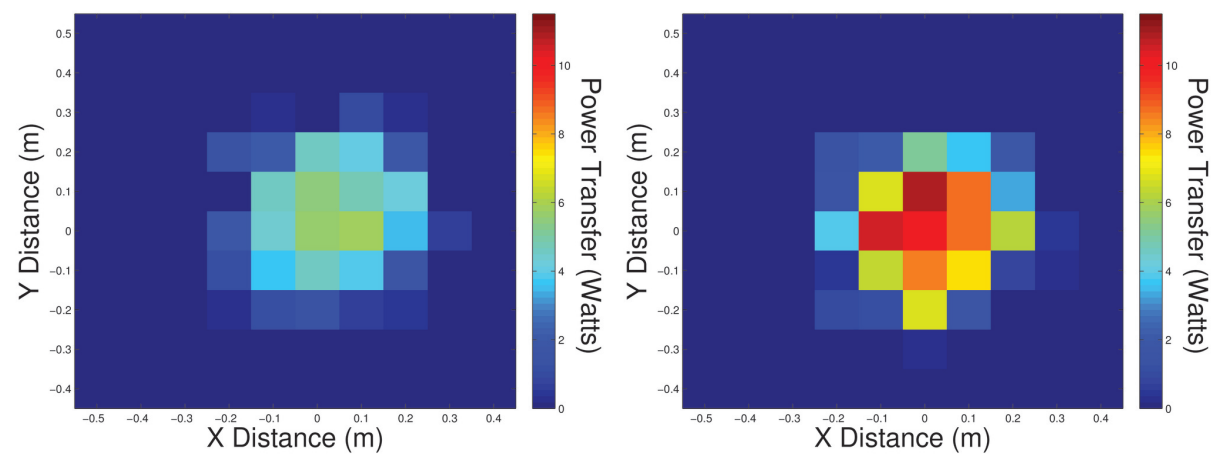

Fig. 5: Power transferred to the sensor node with input voltage of (left) $12 \mathrm{~V}$ and (right) $24 \mathrm{~V}$ recorded at a constant height roughly $5 \mathrm{~cm}$ above the sensor.

Resonant Coil serves to focus the field for transmission to the Rx Resonant Coil, which is placed on the WSN node along with the Load Coil. A magnetic resonant (MR) sensor is connected to the Rx Resonant Coil to detect the Tx system and enable localization. The Load Coil is connected to the receiving board, which draws energy from the Rx Resonant Coil. Finally, the energy from the Load Coil can be stored in the WSN node. Table 1 gives an overview of the specific parameters for the coils we use in our wireless power transfer system.

At the heart of the TX Drive Board is an AD9833 programmable waveform generator that can generate up to a $12.5 \mathrm{MHz}$ signal. This signal is input into an $\mathrm{H}$-Bridge that generates a high-power alternating current that is driven through the Drive Coil. Typically we operate with a 9-24 V input range with a current between 1-4 Amps, although the board is designed to support up to $45 \mathrm{~V}$ and $8 \mathrm{~A}$ with a maximum power rating of up to $100 \mathrm{~W}$. In addition, the TX Board has a processor to control the frequency, enable or disable power transfer, monitor voltage and current, and communicate with the ground sensors and base station with a Zigbee radio.

Fig. 5 shows the amount of power that can be received by the device attached to the load coil using an input of $12 \mathrm{~V}$ and $24 \mathrm{~V}$. This figure shows that there is 

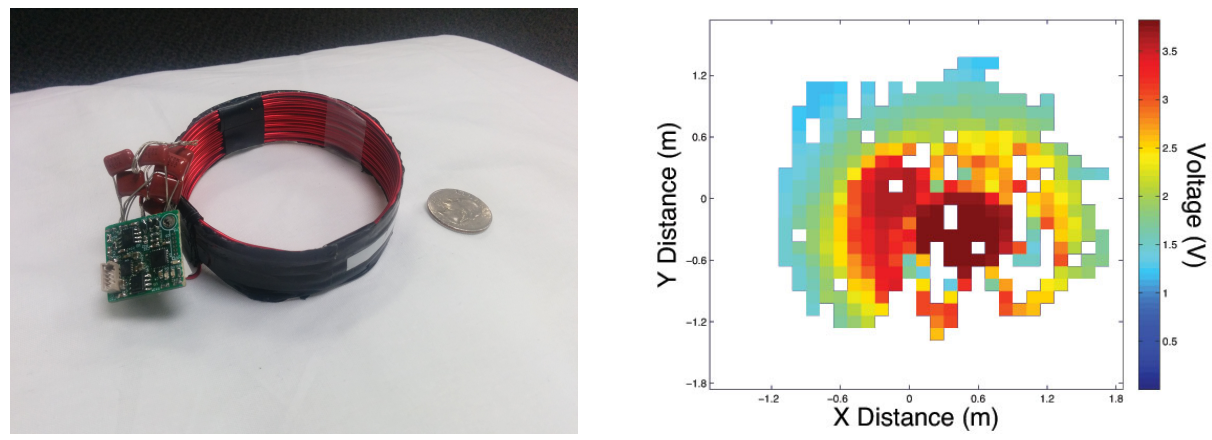

Fig. 6: (left) Magnetic resonant (MR) sensor, which reads the voltage through the resonant coil and (right) a heatmap of the values read $1.0 \mathrm{~m}$ above the sensor (white indicates areas of no data).

a region with about $30 \mathrm{~cm}$ radius which provides over $3 \mathrm{~W}$ of power transfer for $12 \mathrm{~V}$ input (our UAV operating voltage). As the distance from the transmitter to receiver increases past this radius, the amount of power transferred drops significantly. In Section 5 we show that the UAV can consistently localize to within $21 \mathrm{~cm}$ of the sensor. For our applications, $3 \mathrm{~W}$ in a $30 \mathrm{~cm}$ range suffices as this can charge a AAA battery with a single flight, which can power a WSN node for weeks or months. While we are less concerned with optimizing the power transfer system, it is important to note that the overall power transfer can be easily increased. Fig. 5(right) shows that we can double the power transfer, obtaining over $10 \mathrm{~W}$ by doubling the input voltage. This can be accomplished with an additional battery pack attached to the UAV or a UAV with higher operating voltage.

\subsection{Wireless Power Receiver Sensor Node}

A node in the wireless sensor network (WSN) consists of the wireless power receiver board, coils, a magnetic resonant sensor, and any other sensors that are specific to the nodes application, such as vibration, temperature, soil moisture, or pressure sensors. In this paper we omit any application specific sensing system and instead focus on the power transfer system and localizing the UAV onto the WSN node with the wireless power transfer system. A WSN node can receive about $6.1 \mathrm{~W}$ at peak efficiency. With $6.1 \mathrm{~W}$ power transfer for 5 minutes we can nearly charge a typical $\mathrm{NiCd}$ rechargable AAA battery, which can operate most types of low-power sensing systems for weeks. As with the transmitter, there is a Rx Resonant Coil in close proximity to the Load Coil. The receiver board draws energy from the Load Coil and may either use this energy directly or may charge batteries or super capacitors.

A Magnetic Resonant (MR) sensor, Fig. 6(left), is connected to the Rx Resonant Coil and can detect the presence of the UAV power transfer system. When the Tx system approaches, the voltage in this resonant coil increases significantly 


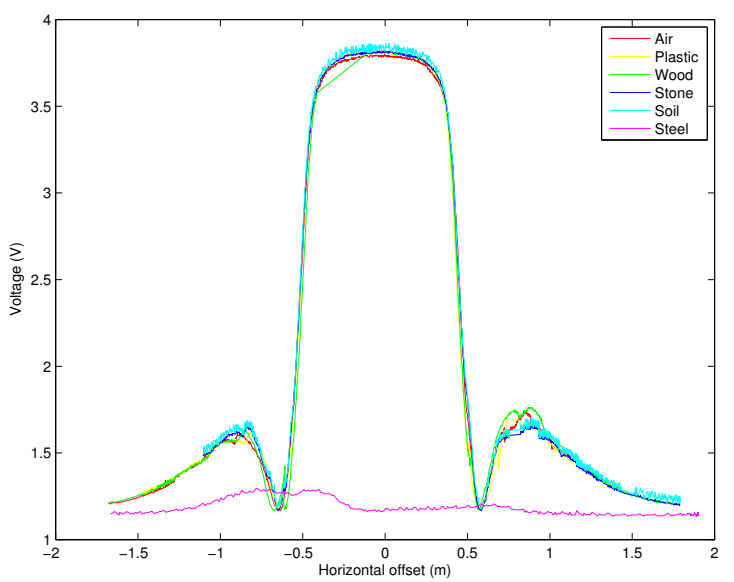

Fig. 7: Voltage readings from an MR sensor which is embedded in different materials.

and is measured by the MR sensor. The advantage of having the MR sensor is that it can detect the power transfer system from three times farther away than the Rx Load Board and is thus the primary input to the localization algorithm. The MR sensor circuit consists of two opamps. The first controls the gain and the second is configured as a precision rectifier to transform the AC signal into a $\mathrm{DC}$ value that is then read by a microcontroller with a 10-bit analog to digital converter (ADC). Fig. 6(right) visualizes the data returned from the MR sensors. As the transmitter approaches the MR sensor, the voltage values approach a maximum value of about $3.7 \mathrm{~V}$. As the distance between the MR sensor and the transmitter increase, the voltage values approach a minimum of around $1 \mathrm{~V}$.

\subsection{MR Sensing Through Different Materials}

We imagine the WSN nodes charged by the UAV may be embedded in many different materials, for example, underground to monitor soil properties. After the UAV has localized the sensor, it can land on the node to maximize charging efficiency (removing the energy required for hovering). In this section, we test the effect of common outdoor materials on the MR sensor.

Fig. 7 compares the voltage through the resonant coil that is embedded in plastic, wood, stone, soil, and steel compared with a baseline measurement of air. The $x$-axis is the horizontal distance, which is circularly symmetric around the MR sensor. The voltage on the $y$-axis is used for localization and is also directly correlated with the power transfer that the WSN node receives.

In this experiment, the WSN node was placed under at least $15 \mathrm{~cm}$ of each of the target materials (except for the steel, where it was covered with a single $1 \mathrm{~mm}$ sheet). The UAV flew a straight horizontal line directly over the WSN node at a constant height of $1 \mathrm{~m}$, and we record the values of the MR sensor. As Fig. 7 shows, the non-ferrous materials have no effect on the reception, however, 
the steel interferes with reception, as would other metals. This means that the sensors can be embedded in many common materials for long-term deployment.

\section{Localization}

In Section 4 we explored the details of the magnetic resonant power transfer system that is attached to the UAV and the WSN node. We showed that the system is able to transfer sufficient power to charge a low-powered WSN node. In this section we address the problem of getting the UAV close enough to the WSN node to transfer power. GPS can record the location of a WSN node when it is deployed, but GPS has up to $7.8 \mathrm{~m}$ error in a $95 \%$ confidence range [19].

Since the UAV must be within $30 \mathrm{~cm}$ to efficiently transfer power, in this chapter we develop a localization algorithm that uses the sensed magnetic field information from the MR sensors to localize over the WSN node. We note that the information regarding intensity is measured by the MR sensor that is located with the WSN node. When the MR sensor first detects power transfer, it turns on its radio and starts broadcasting range information on a short-range radio to the $\mathrm{UAV}$, so little energy is used when the UAV is not charging the node. In addition to using the information from the MR sensor, we also use information from an optical flow sensor on the UAV to provide better dead-reckoning capabilities. Using an optical flow camera for position and control of the UAV means that we add hardware to the UAV, but the WSN node can be significantly smaller. For the experiments presented in this section we simulate an optical flow camera by using a motion capture system with $0.2 \mathrm{~m} / \mathrm{s}$ of Gaussian noise, which is the velocity error reported by the optical flow system developed by Honegger et al. [20].

\subsection{Localization}

In order to estimate the position of the UAV, we must map the voltage information received by the MR sensor to a position in space. First, we can get an estimation of the strength of the magnetic field based on the position of the UAV from an equation shown in [12]:

$$
\bar{B}=\frac{\mu_{0} N I_{i} a}{4 \pi \hat{r}_{i}^{3}}\left[2 \cos (\hat{\theta}) \hat{r_{i}}+\sin (\hat{\theta}) \hat{\theta}\right] .
$$

where $\bar{B}$ is the strength of the magnetic field measured by the MR sensor, $\mu_{0}$ is the magnetic permeability of free space, $N$ is the number of wraps of the coil, $I_{i}$ is the current through the transmitter at time $i, a$ is the area of the coil, $\hat{r}$ is the estimated radial distance from the UAV to the sensor, and $\hat{\theta}$ is the estimated angle from the UAV to the sensor.

The MR sensor provides an ADC value reading from 0 to 600 based on the voltage received by the surrounding magnetic field. These ADC values, $\alpha$, are mapped to the field strength $\bar{B}$ by a lookup table function $\beta(\alpha)$. The lookup 
table is obtained using information similar to Fig. 7 that relates the relative position of the UAV and the MR sensor to the MR sensor reading.

Now that we have mapped the ADC values from the sensor to the field strength, we can develop a localization algorithm for the UAV using range readings from the MR sensor. In order to localize, we must have both the magnetic field strength and a position estimation of the UAV. However, since GPS error is too high, we use an optical flow camera, which can provide accurate motion estimates over short periods of time [21,22] with higher accuracy than GPS.

The particular optical flow camera we attempted to use in our system (PX4Flow [21]) was very sensitive to its viewing surface. We tested many surfaces and found a minimum error of $0.2 \mathrm{~m}$ over a $10 \mathrm{~s}$ flight over a textured wood surface (similar to that reported by the developers), but very poor results with other materials. While the best error more than acceptable, we could not obtain enough material to cover the entire testing area. Therefore, we simulate the optical flow camera by introducing ten times as much noise $(0.2 \mathrm{~m} / \mathrm{s})$ into the speed estimation of the UAV.

The MR sensor is placed directly on the receiving resonant coil of the WSN node. We then use a least squares approach to find the location of the WSN node. We do this by attempting to find a location $\left(\hat{x}_{s}, \hat{y}_{s}\right)$ which minimizes the difference between the position of the UAV and the range measurements from the MR sensor. Specifically, we minimize the function:

$$
\underset{\hat{x}_{s}, \hat{y}_{s}, \hat{\theta}}{\arg \min } \sum_{i=1}^{n}\left(\frac{\mu_{0} N I_{i} a}{4 \pi \hat{r}_{i}{ }^{3}}\left[2 \cos (\hat{\theta}) \hat{r_{i}}+\sin (\hat{\theta}) \hat{\theta}\right]-\beta\left(\alpha_{i}\right)\right)^{2}
$$

where ${\hat{r_{i}}}^{2}=\left(\hat{x}_{u a v_{i}}-\hat{x}_{s}\right)^{2}+\left(\hat{y}_{u a v_{i}}-\hat{y}_{s}\right)^{2},\left(\hat{x}_{u a v_{i}}, \hat{y}_{u a v_{i}}\right)$ is the estimated position of the UAV given by the simulated optical flow, $d_{i}$ is the distance measurement from the MR sensor that corresponds with that UAV position, and $\hat{z}_{s}=\hat{z}_{\text {uav }_{i}}-\hat{r}_{i} \sin \hat{\theta}$ is the height of the sensor.

Squaring the value means that the closer the UAV is to the receiver, the higher the weight is given to the function. This means that high voltages will penalize the function value much more for incorrect arguments.

We then find the estimated position of the MR sensor, $\left(\hat{x}_{s}, \hat{y}_{s}\right)$, that minimizes this function over the $n$ readings. The data used for input to the minimization function is in the structure of a circular queue that holds up to 10 seconds of data. Allowing this temporal property prevents the minimization function from becoming overweighted with areas with dense sampling, producing erroneous position estimates from too few samples, and problems from longer-term optical flow position estimation drift.

Minimizing Eqn. 3 produces accurate position estimates as long as there are sufficient samples within $1 \mathrm{~m}$ of the MR sensor. Alg. 1 shows the algorithm we use to ensure good sampling of the area. The localization algorithm works by first approaching the position of the sensor that was recorded during deployment using GPS alone. The UAV will be near the sensor when it arrives at its coarse position, but not close enough to be able to transfer power to the sensor. Immediately after a non-zero reading from the MR sensor is encoutered, the UAV will 


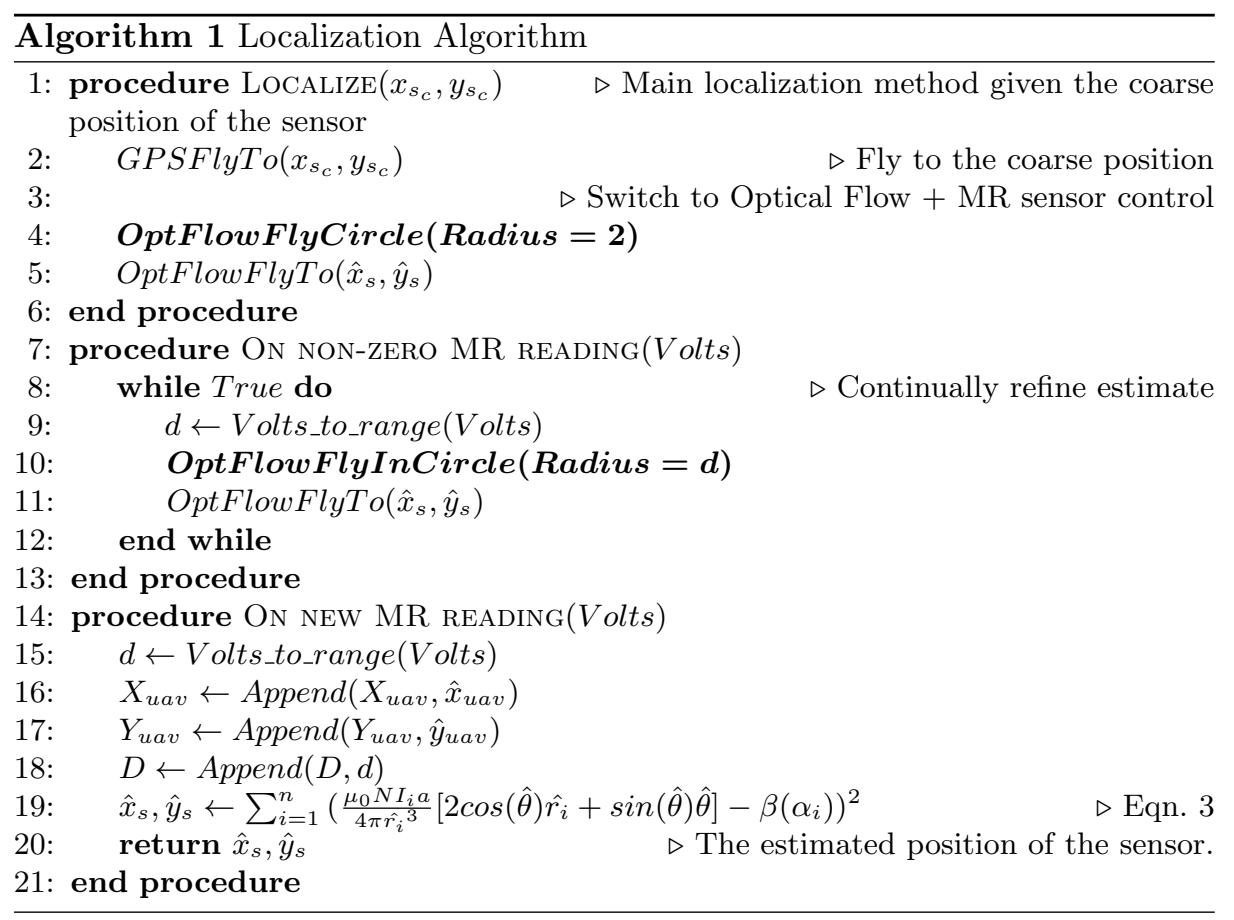

perform a circular trajectory with radius equal to the range estimation that it has received. Once the circle is complete, the UAV flies to the estimated position of the WSN node (line 5), which is computed in a separate thread continuously (lines 11 through 17). This procedure is performed in a loop to continually refine the estimate (lines 6 through 8 ) until some other action is taken (for example, land, fly home, fly to another WSN node).

We performed over 10 localization trials using this approach. On average, the UAV localized with an average error of $15 \mathrm{~cm}$, with a $6 \mathrm{~cm}$ standard deviation and an average localization time of $46 \mathrm{~s}$. Fig. 8(left) shows the UAV path localizing the sensor with this method. Fig. 8(right) shows the range readings compared to ground truth (obtained with a motion capture system) and the power transfer rate. During time $t=0$ to $t=5$, no valid range estimate had been found, thus there is no estimated distance. The UAV continued the scripted flight until $t=15$, when it then flies to the current estimate of the sensor's position. In this particular experiment, the position was found within $6 \mathrm{~cm}$ of the true location after 24 seconds. At this distance, the WSN node receives $5.49 \mathrm{~W}$ and the MR sensor is at $100 \%$ of its maximum value.

\section{Conclusion}

The UAV-based wireless power transfer system presented in this paper allows sensors in remote locations to be recharged autonomously. We discussed design 

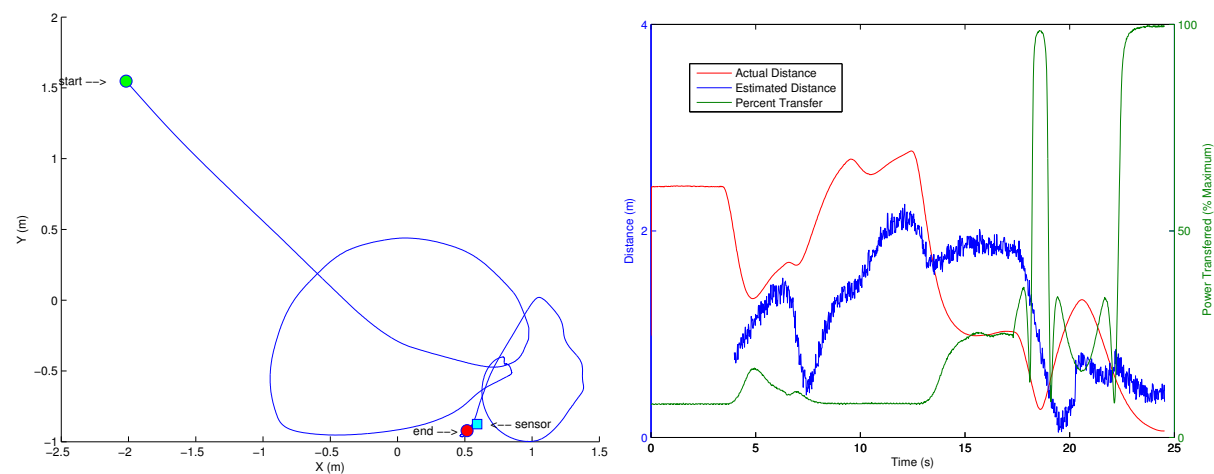

Fig. 8: (left) Localization path and (right) true distance, estimated distance, and power transfer from a localization experiment.

considerations for UAV-based wireless power transfer systems and presented the details of our system. We showed that many common outdoor materials (wood, dirt, stone, soil) have little impact on the power transfer system We have presented a unique localization strategy that measures the magnetic field that is emitted from a resonant wireless power transfer system. The localization strategy allows the UAV to localize a WSN node with higher precision than is available with GPS. The UAV is able to localize to an average of $15 \mathrm{~cm}$ to land on the WSN node and charge it with an average of $4.2 \mathrm{~W}$. This shows that it is possible for a UAV charge sensors deployed in remote and hard to access locations.

\section{Acknowledgments}

This work was partially supported by NSF CSR-1217400 and USDA NIFA National Robotics Initiative 2013-67021-20947. Any opinions, findings, and conclusions or recommendations expressed in this material are those of the authors and do not reflect the views of the sponsors.

\section{References}

1. R. Szewczyk, A. Mainwaring, J. Polastre, J. Anderson, and D. Culler, "An analysis of a large scale habitat monitoring application," in Proceedings of the Conference on Embedded Networked Sensor Systems, pp. 214-226, 2004.

2. I. F. Akyildiz, Z. Sun, and M. C. Vuran, "Signal propagation techniques for wireless underground communication networks," Physical Communication, vol. 2, no. 3, pp. 167-183, 2009.

3. C. Detweiler, S. Banerjee, M. Doniec, M. Jiang, F. Peri, R. F. Chen, and D. Rus, "Adaptive decentralized control of mobile underwater sensor networks and robots for modeling underwater phenomena," Journal of Sensor and Actuator Networks, vol. 3, no. 2, pp. 113-149, 2014. 
4. B. Griffin and C. Detweiler, "Resonant wireless power transfer to ground sensors from a uav," in Robotics and Automation (ICRA), pp. 2660-2665, 2012.

5. J. O. McSpadden and J. C. Mankins, "Space solar power programs and microwave wireless power transmission technology," Microwave Magazine, vol. 3, no. 4, pp. 46 57, 2002.

6. A. Sample and J. R. Smith, "Experimental results with two wireless power transfer systems," in Radio and Wireless Symposium, pp. 16-18, 2009.

7. N. Tesla, "Apparatus for transmitting electrical energy," 1914

8. A. Kurs, A. Karalis, R. Moffatt, J. D. Joannopoulos, P. Fisher, and M. Soljačić, "Wireless power transfer via strongly coupled magnetic resonances," science, vol. 317, no. 5834, pp. 83-86, 2007.

9. T. Duong and J. Lee, "Experimental results of high-efficiency resonant coupling wireless power transfer using a variable coupling method," Microwave and Wireless Components Letters, vol. 21, no. 8, pp. 442-444, 2011.

10. A. P. Sample, D. A. Meyer, and J. R. Smith, "Analysis, experimental results, and range adaptation of magnetically coupled resonators for wireless power transfer," Transactions on Industrial Electronics, vol. 58, no. 2, pp. 544-554, 2011.

11. Y.-S. Seo, Z. Hughes, M. Hoang, D. Isom, M. Nguyen, S. Rao, and J.-C. Chiao, "Investigation of wireless power transfer in through-wall applications," in Microwave Conference Proceedings (APMC), pp. 403-405, 2012.

12. J. Moore and R. Tedrake, "Magnetic localization for perching uavs on powerlines," in Intelligent Robots and Systems (IROS), 2011 IEEE/RSJ International Conference on, pp. 2700-2707, IEEE, 2011.

13. P. Tokekar, D. Bhadauria, A. Studenski, and V. Isler, "A robotic system for monitoring carp in minnesota lakes," Journal of Field Robotics, vol. 27, no. 6, p. 779789, 2010 .

14. W. C. Brown, "The history of power transmission by radio waves," Transactions on Microwave Theory and Techniques, vol. 32, no. 9, pp. 1230-1242, 1984.

15. M. C. Achtelik, J. Stumpf, D. Gurdan, and K.-M. Doth, "Design of a flexible high performance quadcopter platform breaking the mav endurance record with laser power beaming," in International Conference on Intelligent Robots and Systems (IROS), pp. 5166-5172, 2011.

16. O. Bishop, Electronics - Circuits and Systems. Taylor \& Francis, 2012.

17. B. Griffin, "Automated resonant wireless power transfer to remote sensors from an unmanned aerial vehicle," Master's thesis, University of Nebraska - Lincoln, Lincoln, NE, 2012.

18. A. Karalis, J. D. Joannopoulos, and M. Soljačić, "Efficient wireless non-radiative mid-range energy transfer," Annals of Physics, vol. 323, no. 1, pp. 34-48, 2008.

19. N. C. O. for Space-Based Positioning Navigation and Timing, "Global positioning system standard positioning service performance standard 4e," science.

20. D. Honegger, P. Greisen, L. Meier, P. Tanskanen, and M. Pollefeys, "Real-time velocity estimation based on optical flow and disparity matching," in International Conference on Intelligent Robots and Systems (IROS), pp. 5177-5182, 2012.

21. F. Kendoul, I. Fantoni, and K. Nonami, "Optic flow-based vision system for autonomous 3d localization and control of small aerial vehicles," Robotics and Autonomous Systems, vol. 57, no. 6, pp. $591-602,2009$.

22. S. Weiss, D. Scaramuzza, and R. Siegwart, "Monocular-slambased navigation for autonomous micro helicopters in gps-denied environments," Journal of Field Robotics, vol. 28, no. 6, pp. 854-874, 2011. 